\title{
Damage and Management of Alien Species in China
}

\author{
Xidong Mu, Yinchang Hu (Corresponding author), Hongmei Song, Peixin Wang \& Jianren Luo \\ Pearl River Fishery Research Institute, Chinese Academic of Fishery Science \\ Guangzhou 510380, China
}

Tel: 86-20-221-75232Ｅ-mail: muxd1019@163.com

\begin{abstract}
This study was supported by Agricultural Biological Resources Protection and Utilization Project(No. 2130108) and the Open Fund of Key Laboratory of Genetic Breeding and Aquaculture Biology of Freshwater Fishes, Ministry of Agriculture( No. BZ2009-10).
\end{abstract}

\begin{abstract}
Alien invasive species have been a seriously environment issue in the whole world. The ecological damages and economic influences of alien invasive species are analyzed in this article, and the government is suggested to strengthen the quarantine services, strictly manage the intentional introduction of alien species, and implement the risk supervision system for the introduction of alien species.
\end{abstract}

Keywords: Alien species, Damage, Management

\section{Definition and influence of alien species}

Alien species mean the species which originally don't exist in the region or the ecological system, but are introduced because of human activities or relative causes about human activities. The alien species from international countries are called as the foreign alien species, and the alien species from the different regions in same one country are called as the regional alien species, and the corresponding specials are called as the native species (Li, 2002, P.211). When alien specials enter into a new region, survive and propagate, and form a feral population which further diffuses, and has formed or will form ill ecological or economic effects, and this event is called as the biological invasion, and the alien species induced the biological invasion are called as the alien invasive species. As viewed from human history, the probability that alien species invaded the local ecological environment was very small, and "the probability that one species becomes the invasive species is only about 1\%o (Lu, 2003, P.35-37)". But with the increasing frequency of international economic communication and the convenience of the traffic, the gradual elimination of original geographic factor, the communication and penetration of species among different regions in the world become more and more frequent, and the invasive risk of alien species increases at the same time (Naylor RL, 2001, P.1655-1656). Alien species usually invade local ecological environment by two approaches, i.e. the artificial factor and the natural factor, and the artificial factor is divided into the unintentional introduction and the intentional introduction, and the intentional introduction has been the main approach that alien species invade China at present. According to the incomplete statistics, the alien invasive ruderals to China are 108 sorts, and 62 sorts of them are intentionally introduced, $58 \%$ of the total amount (Cai, 2003, P.27-34). The alien invasive species will induce very serious damages to the human being and the society, which are mainly embodied in the ecology and the economy.

\subsection{Ecological influences}

\subsubsection{The invasion of alien species will induce the loss of the biological diversity in the ecological system}

In each one special ecological system, because of the long-term evolution of the nature, biology restrains their natural enemies each other, and is influenced by other factors such as climate, and each population is limited in the special region and quantity. When one species enters into one new region, it will easily become the dominant species because of various causes (such as lacking in sufficient biological resistance) to reduce the species diversity, and make other survived species depending on the local species diversity to lose the comfortable habitat. For example, Christian thought that the reciprocity relation between the seed spreader and the plants in the "animal-plant-seed spread" system was very important to maintain the survival of the species, update the community structure and the diversity in his "Hypothesis of Key Mutual Benefits". The loss of certain one species induced by the invasion of alien species will induce the chained extinguishing disaster of relative species (Christian, 2001, P.635-639). 
1.1.2 The invasion of alien species will induce the loss of the genetic diversity in the ecological system and serious biologic genetic pollution

Invasive species will contest with indigenous species for survival space and foods, occupy the ecological niche of local species, and threaten the survival of indigenous species. Invasive species will also directly kill local species (such as eating the ovum, juveniles even adults of local species) to influence the survival of local species, or directly threaten local species, or excrete and release chemical substances to reduce the sorts and quantity of local species and restrain the growth of other species, even make them to be in severely ill or killed out, which will induce the loss of the genetic diversity of the ecological system. Invasive species will also hybridize with indigenous species with close relative, which may induce the genetic erosion of the later, and some genes which can not suit for local environment may be inherited to indigenous species, and the inheritance quality of indigenous species may be reduced, and the genetic pollution will occur, and the genetic diversity of the ecological system will be impacted, so the biological diversity of the ecological system will be reduced.

\subsubsection{The invasion of alien species will induce the decrease and loss of the protection ability of the ecological system}

Because of the invasion of alien species, many alien invasive species began to destroy the local vegetation, and form the mono-dominant community, and indirectly reduce the sorts and quantity of other local species depending on the local vegetation, and finally the singularity and degeneration of the ecological system will form, and the ability of the biology which resist exterior risks depending on the species diversity in the complex ecological system and the biologic chain will gradually reduce. Invasive species may also bring causal organisms and parasites which will destructively attack indigenous species, and largely damage the local ecological environment, and they impact the species structure and the food web structure of the local ecological system by preying and competition, so the capacity to withstand risks of the ecological system will be reduced.

\subsection{Economic influences}

Alien invasive species are integrated pests which will directly harm the development of forest economy, and the quick diffusion of alien invasive species may quickly change the natural sight of the ecological system, such as covering rivers and lakes, destroying forests and grasslands, and harming environment and human safety, making the original tourism region to lose value and bringing loss to the tourism industry. Alien species could produce indirect economic loss by a series of bad influences on water and soil and climate changing the ecological system. According to the first alien invasive species survey report in China, there were total 283 sorts of alien invasive species which would produce 119.876 billion Yuan of direct or indirect economic loss, 1.36\% of GDP (Xu, 2004, P.138-147 \& 166-181), and only several sorts of main alien invasive species would induce 57.4 billion Yuan of economic loss in each year (Li, 2002, $1-9)$.

At the same time, the ecological effects and economic influences of same one species may be different even completely different because of the difference of time and place. Most introduced alien species are beneficial for human beings, and one species, one variety even one gene may be the headspring to flourish the economy of the country (Xue, 2005, P.585), such as the Tilapia and penaeus vannamei boone. When alien species enter into the new environment, they should possess certain internal and external reasons to become new dominant species. According to Williamson's theory of "tens digit law" (Williamson, 1996, P.1664-1666), once alien species invade the local environment successfully, it is very difficult to fully eliminate their influences, and the costs controlling their damages and diffusions are very large by using large numbers of financial, material and human resources. Therefore, the governments should adopt various effective measures to prevent the possibility of the invasion of alien species.

\section{Countermeasures to prevent the invasion of alien species}

Aiming at many deficiencies and shortages existing in the legislation about the present alien species invasion of China, the innovation of legal system is the base and key to establish the legal prevention system of alien species invasion. Various parts of the invasion of alien species such as establishing relative legal management measures aiming at each one invasion approach and ensuring the coherence of relative legal articles with corresponding international convention and agreements should be fully considered to completely realize the management of alien invasive species by law.

\subsection{Prevention of the "unintentional introduced" species}

According to the research and survey report of the key project aiming at the invasion of alien species, $76.3 \%$ alien invasive animals entered China with the trading goods or the conveyance because of loose checking (Ou, 2006, P.1240-1244). And the international commercial trading and the travel of tourist are always the convenient approach for the invasion of alien species (Qin, 2004, P.44-47), for example, the golden-rod of Canada was unintentionally carried by tourists and entered China ( $\mathrm{Li}, 2003$, P.7-9). Once the checking is loose, such convenient ports and land traffic may intentionally or unintentionally introduce more alien species, and a few of them may induce large damages. Therefore, the import quarantine supervision should be strengthened to prevent the introduction of alien harmful species and reduce the quantity of introduced harmful species to the lowest (Chen, 2005, P.49-50). 
Following countermeasures and advices are suggested, (1) establishing the alien invasive species identification and alarming information center, and building the database and information system of the harmful invasive species to college, dispose and feed back relative information and results, (2) strengthening the construction of the ability of alien species risk evaluation, and analyzing the risks of potential invasive species, and perfect the quarantine and the environment supervision measures of alien invasive species, (3) strengthening the relative scientific researches to preventing the invasion of alien species, and closely associating with the institutions which want to intentionally introduce alien species, and cultivating the public's consciousness and conscious behaviors to face the invasion of alien species by various medias and channels.

\subsection{Prevention and management of the invasion of "intentional introduced" species}

Relative systems should include the ecological safety risk evaluation system before the introduction of species, the emergence reaction system of harmful invasive species, the ecological safety supervision system of introduced species and the elimination system of invaded species, and the prevention, evaluation and supervision of the species invasion.

\subsubsection{Establishing the catalog system of species and the risk supervision system}

The article 14 of the "National Ecological Environment Protection Program" specially regulated that "the introduction of alien species must be assessed by risk", because the damage of the alien invasive species is very large, and once the damage forms, the possibility of elimination is very small, so it is very important to study which species will be the invasive species, where the invasion will induce damages and what damage will be induced (Xu, 2003, P.4-8). The main points and difficult points of the alien species risk evaluation are the establishment and design of the index system (Ding, 2006, P.92-96), and according to the varieties, introduced approaches, the biological and ecological characters, and the difference of damage characters of alien species, different ecological safety risk evaluation systems (respectively aiming at terrestrial pants, land invertebrate species, aquatic invertebrates, microorganisms, aquatic plats, and aquatic animals) and different licensing systems and access systems with different classes should be established. Because of large differences of the damage states in different ecological systems of alien species, the alien species invasive risk evaluation index system for special region should be established and different species should have different evaluation indexes. The present Weed Risk Assessment System of Australian (Chen, 2001, P.466-471) is composed according to a series of question arsenal including the ecological characters, geographic characters, and biological characters of the species. Agreement on the Alien Pants Evaluation of Hawaii established by Daehler et al (Daehler C C, 2004, P.360-368) is mainly to evaluate the risks of the possible invasive alien plants to Hawaii and other Pacific islands from four aspects including "the influence of invasive species on the ecological system, the living character of species, the potential diffusion ability and the control difficulty". Jiang Qing et al (Jiang, 1994, P.331-334) put forward the harmful species fatalness evaluation index system, Ji Liang et al (Ji, 2004, P.100-105) analyzed the fatalness of quarantine harmful biology, and Xiang Yanci et al (Xiang, 2002, P.40-48) made the ecological risk evaluation and management of alien plants, and the PRA Project Group of "the Eighth Five-Year Plan" of China Ministry of Agriculture had confirmed the harmful biology fatalness evaluation system in 1997. Yu Yinchang et al (Hu, 2006, P.113-115) put forward the alien aquatic animal invasion risk evaluation system according to the fertility, the competitive strength and the viability of aquatic animals.

In conclusion, the invasion of alien species is a complex chain process, and it is a long-term process from colonization, constructive species to diffusion, and many invasive species have a time-lagging term after introduced, and different species have different time-lagging terms such as a few years even tens of years. The damage of alien species to the genetic diversity is irreversible (Liu, 2004, P.94-98). Therefore, the environmental risks of alien species should be evaluated, and only those species with few or small influences on the ecological environment after the environment risk evaluation can be introduced.

\subsubsection{Establishing the risk prevention system}

First, check the actuality of alien species clear. The detailed information of the alien species in China, such as the invasion varieties, the source areas, the distribution areas and the transmission routes, should be made clear to study the control measures and establish the supervision system aiming the existing invasive species or potential invasive species. Based on that, the prevention and comprehensive treatment of known alien harmful species should be strengthened. Second, confirm the key prevent area of main alien invasive species, and relative departments should confirm and publicize above information. Local governments should strengthen the environment management in the natural reserves, the landscape and famous sceneries, and forest parks to prevent the intentional or unintentional introduction of alien invasive species. Third, strengthen the scientific research and enhance the level of environment management. For the prevention and treatment of alien invasive species, the technology is the first measure. At present, many countries in the world all very pay attention to the communication in this technical domain, and China should establish the integrated management system including ecological system, habitat restoration, biological prevention, low-pollution prevent, ecological replacement, early earning and remote sensing by exterior species environment influence evaluation and foreign alien species control technology. 


\subsubsection{Establishing the species introduction supervision system}

Local governments should establish the stable breeding and representative area system, i.e. in the planned land, adopting the stable breeding measure to the introduced species with uncertain harm in 1 2 years, and supervising their growth and propagation, and creating profiles for these species, especially for those species with strong suitability and fecundity. At the same time, set up the buffer zones surrounding of the breeding area, which can help to control the invasion of alien species. The spread of introduced species must be demonstrated by relative experts and evaluated by relative institutions. The good quick response system aiming at the invasion of alien species should be established, and once alien species have invaded the region or have the threat to invade the region, the relative department should adopt proper measures such as eliminating, restraining and controlling as soon as quickly to reduce the negative influences. For the confirmed alien invasive species, or the alien invasive species which have been controlled, or the alien introduced species with higher hazard index, relative department should strictly supervise them to prevent their spread or repeated invasion. For the alien invasive species (such as Pomacea canaliculata and Cambarus proclarkii) which have induced harms, the comprehensive technology measure should be studied and developed to reduce or eliminate their damages, and for the alien invasive species (such as Brazilian Slider and ashman) with potential harms, relative department should strictly restrain them in the breeding range, and adopt necessary measures to prevent escaping and limit the breeding scale, and implement strict track supervision to eliminate the dangerous alien economic animals in the initial diffusion stage.

\section{References}

Cai, Lei, Yu, Zhidi \& Wang, Jie. (2003). Control Alien Invasive Species to Conserve Biodiversity. Environmental Protection, No. 8, P. 27-34.

Chen, Liangyan \& Xu, Haigen. (2001). Australian Management Strategy for Alien invasive species and References Available to China. Biodiversity Science, No. 9(4), P. 466-471.

Chen, Wentian. (2005). Environment Risk Evaluation of Alien Species. Perspectives of Scientific and Technological Achievement. No. 3, P. 49-50.

Christian C E. (2001). Consequences of a biological invasion reveal the importance of mutualism for plant communities. Nature, No. 413, P. 635-639.

Daehler C C, Denslow J S \& Ansari S, et al. (2004). A risk assessment system for screening out invasive pest plants from Hawaii and other Pacific Islands. Conserv. Biol., No. 18, P. 360-368.

Ding, Hui, Shi, Biqing \& Xu, Haigen. (2006). Index System and Methodology for Risk Assessment of Alien Species. Journal of Ecology and Rural Environment, No. 22(2), P. 92-96.

$\mathrm{Hu}$, Yinchang, Li, Yong \& Luo, Jianren et al. (2006). Risk Assessment System for Alien Aquatic Animals. Journal of Huazhong University of Science and Technology (Nature Science), No. 36(10), P. 113-115.

Ji, Liang. (2004). Evaluation of Quarantine Harmful Biology Fatalness. Plant Quarantine, No. 8(2), P. 100-105.

Jiang, Qing, Liang, Yibing \& Wang, Naiyang et al. (1994). Primary Confirmation of Harmful Biology Fatalness Evaluation Index System. Plant Quarantine, No. 8(6), P. 331-334.

Li, Deqiu. (2003). Prevention against Alien Pest Species. Guangdong Forestry Surveying \& Designing, No. 4, P.7-9.

Li, Zhenyu \& Xie, Yan. (2002). Alien Invasive species of China. Beijing: Chinese Forestry Press. P. 211 \& 1-9.

Liu, Pengcheng. (2004). It is of Great Urgency for Prevention from Adventitious Biological Invasion. Forest Inventory and Planning, No. 29(2), P. 94-98.

Lu, Ruisuo \& Song, Yuqin. (2003). Reasons of Biological Invasion in Lakes of Yungui Plateau. Environmental Protection, No. 8, P. 35-37.

Naylor RL, Williams SL \& Strong DR. (2001). Aquaculture-A gateway for exotic species. Science, No. 294(23), P. $1655-1656$.

Ou, Jian \& Lu, Changyi. (2006). Present Situation of Alien Plants Invasion and Its Risk Assessment System in Xiamen City. Chinese Journal of Ecology, No. 25(10), P. 1240-1244.

Qin, Datang \& Cai, Bofeng. (2004). Risk Assessment of Biological Invasion in Beijing. Environment Protection, No. 1, P. 44-47.

Williamson M \& Fitter A. (1996). The varying success of invaders. Ecology, No. 77, P. 1664-1666.

Xiang, Yanci \& Peng, Shaopin \& Ren, Hai et al. (2002). Management and Ecological Risk Assessment of Exotic Plants. Chinese Journal of Ecology, No. 21(5), P. 40-48.

Xu, Haigen \& Wang, Jianmin. (2004). New Hot Research about "the Convention on Biological Diversity": Biological 
Safe Inheritance Resources of Alien Invasive Species. Beijing: Science Press, P. 138-147 \& 166-181.

$\mathrm{Xu}$, Rumei. (2003). Data Integration, Quantity Analysis and Alarm of Biological Invasion. Beijing: Science Press. P. 4-8.

Xue, Dayuan. (2005). Actuality and Protection of Chinese Biological Inheritance Resources. Beijing: China Environmental Science Press. P. 585. 